\title{
Rectify Malignant and Benign Tumor cancer by Digital Image Processing Techniques
}

\author{
Navjot Kaur \\ Department of computer science and Engineering \\ IET BHADDAL, Ropar, Punjab, India \\ Dr. Sanjay Singla \\ Department of computer science and Engineering \\ IET BHADDAL, Ropar, Punjab, India
}

\begin{abstract}
Breast cancer, the second common cancer after lung cancer realized in females if compared with males.GLCM features are are energy, correlation, contrast and homogeneity. In this proposed work,features which realized by GLCM called Gray Level Co-Occurrence Matrix and comparison between the features having original image values and their corresponding images values having cancer are done. DDSM) and MIAS images are used to expose the benign and malignant cancer by various digital image processing techniques. For classification of Malignant and Benign cancer,SVM Classifier has been used as having good accuracy.
\end{abstract}

IndexTerms-Malignant,Benign,DDSM/MIAS,GLCM Features,SVM Claasifier.

\section{INTRODUCTION}

Malignant and Benign cancer are the most common cancer after the lung cancer.These cancers are the types of breast cancer.GLCM,2nd order statistics, is used to extract the features. .Haralick put forward for considerations different features. These features are calculated at separate angles. These angles are at $[1,-1],[-1,0],[0,1],[-$ 1,1].GLCM order texture measures are First order texture features ,Second order texture ,Third and higher order textures features .[2]GLCM is calculated from gray scale image values only. It cannot be applied on RGB images.

SVM Classifier is used to detect whether the benign or malignant class.KNN Classifier is also used to detect the class of cancer. But SVM Classifier is good as compared to KNN Classifier. There are Different types of filters which are used to remove the noise. the filters are imfilter, median filter and Gaussian filter. On comparison of these median filter gives the good result as compared to other filters.

1. Imfilter: This filter is used to remove the noise but the image is not cleared as median filter.This filter is used on three dimensional RGB images. Input images do not convert into double with this filter. The result is shown below:

2. Gaussian Filter: This filter has the same function as the imfilter but this also not give the good result as median filter. It has function to blur the image. It also uses the standard deviation function. The result of this filter is given below:

3. Median Filter: This filter is non linear digital filter and the operators are used with this filter.the operators can be sobel, prewitt operator etc. This filter is used more frequently than imfilter and Gaussian filter because the image is more clear with this filter than other filters. The result is shown below: 


\section{FLOW CHART:}

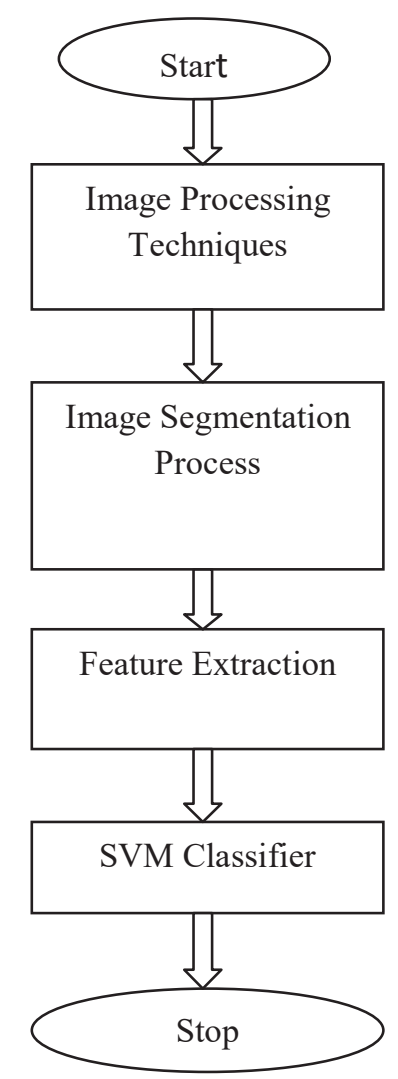

1.1 SVM Classifier: SVM is called the Support Vector Machine and this classifier is used to classify the images which depicts that which portion of the image is malignant or benign.It is effective for higher dimensional images. The cases when samples are less than the number of dimensions this classifier is also operative .Its accuracy is good than KNN classifier.SVM Classifier is used to classify the all pixels of the image.This depicts that this division of the image are of two regions and the boundary between these two regions is called the optimal separating hyperplane.This hyperplane is used to differentiate between two classes whether the cancer is malignant or benign. In this Support Vector classification, the function which is used is a combination of kernels linearly associated with the Support Vectors. This formulation has advantage that the SVM training is required for solving a problem having quadratic optimization which is used for optimization routines from libraries having numerical values. This step is fast computationally and problem regarding stability is there [5]. Other Attractive iterative algorithms i.e. the Sequential Minimal Optimization ( SMO ), Nearest Point Algorithm ( NPA ) etc. are there to defeat this problem [4], [5]. SVM Classifier has accuracy of 95\% approximately.

\section{PROCEDURE}

1.The original image is taken. The image can be Digital database for screening mammography or The Mammographic Image Analysis Society .

2.The DDSM/MIAS image is changed into the form into Gray Scale as GLCM Features extract on gray scale images only.

3.Cropping method is used to remove the noise like artifacts. It is optional as no artifact is present.

4.Filtering is done and filter used here is median filter as filteration is good than others. imfilter filter is also used here to observe the comparison between median filterd image and imfiltered image.

5.Thresholding is the another process which is used on filtered image.By using thresholding binary image can get. 
6.Segmenttaion is the another process which is used after thresholding of the image that segments the threshold image.[4],[5]

7.Classifier is the main requirement to classify whether the cancer is malignant or benign.SVM Classifier is used to classify the image as its accuracy is good than KNN classifier etc.

Here PN is used for Patient.The results of the various images are:

A. IMAGE DDSM (PNT1)

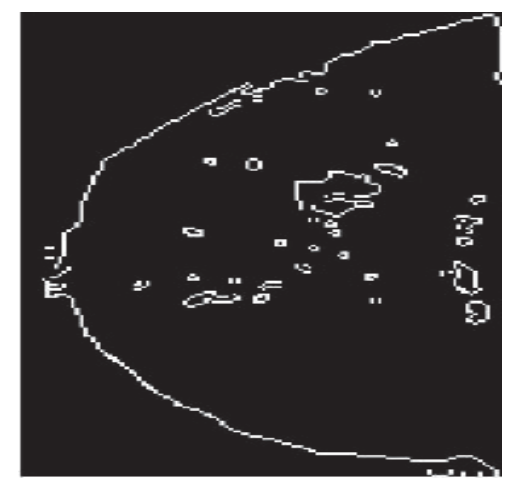

Fig 1 Segmented Image

B. IMAGE DDSM (PNT2)

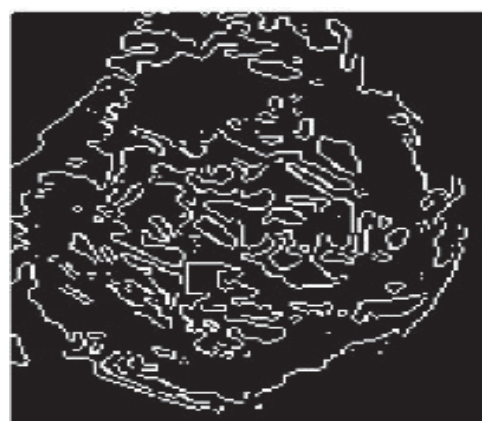

Fig 2(af)Segmented Image

Table 1: GLCM Texture Features Values

\begin{tabular}{|c|c|c|}
\hline $\begin{array}{c}\text { Texture } \\
\text { Features }\end{array}$ & $\begin{array}{c}\text { PNT1 } \\
\text { CV }\end{array}$ & $\begin{array}{c}\text { PNT2 } \\
\text { CV }\end{array}$ \\
\hline Offset[0 1] & & \\
\hline Contrast & 0.4869 & .7626 \\
\hline Correlation & 0.842 & .9588 \\
\hline Energy & 0.9275 & .4865 \\
\hline Homogeneity & 0.9923 & .9864 \\
\hline Offset[-1 1] & & \\
\hline
\end{tabular}




\begin{tabular}{|c|c|c|}
\hline Contrast & 0.9866 & 1.0501 \\
\hline Correlation & 0.7014 & .9570 \\
\hline Energy & 0.9176 & .4809 \\
\hline Homogeneity & 0.9843 & .9812 \\
\hline Offset[-1 0] & & \\
\hline Contrast & 0.4983 & .7403 \\
\hline Correlation & 0.8881 & .9697 \\
\hline Energy & 0.3965 & .4768 \\
\hline Homogeneity & 0.9921 & .9878 \\
\hline Offset[-1-1] & & \\
\hline Contrast & 1.1906 & .9596 \\
\hline Correlation & 0.7575 & .9604 \\
\hline Energy & 0.8925 & .4823 \\
\hline Homogeneity & 0.9804 & .9837 \\
\hline
\end{tabular}

CV-Cancerous Image Value PNT-Patient

\section{CONCLUSION}

It was noticed that the features of GLCM are calculated and Benign and malignant cancer can be found by using SVM Classifier and this classifier has ability to classify the two classes malignant and benign. The features are extracted by using GLCM.

\section{REFERENCES}

[1] http://www.fp.ucalgary.ca/mhallbey/what is texture.html

[2] http://matlab.izmiran.ru/help/toolbox/images/enhanc15.html

[3] Blaine Martinez," Digital Image Processing Final Project" and Nicholas Sia Pik Kong, Theam Foo Ng, "Simple Adaptive Median Filter for the Removal of Impulse Noise from Highly Corrupted Images Haidilbrahim", IEEE Transactions on Consumer Electronics, Vol 54, No.4 ,Novermber 2008

[4] Robert M. Haralick ,K. Shanmugam and Its'hak Dinstein,’Textural Features for Image Classification”, IEEE Transcations on systems,man and cybernetics,vol.SMC-3 pp. 610-621,6-Nov 1973

[5] Pawar B.V., Patil Sharma,"Early Stage Detection ofTumors in Mammograms",Electronic Dept.,W.C.E. Sangli.

[6] Aswini Kumar Mohanty, Swapnastikta Beberta,S aroj Kumar Lenka," Classifying Benign and Malignant Mass using GLCM and GLRLM based Features from Mammogram", International Journal of Engineering Research and Applications,col.1,pp.6876-693

[7] P. Mohanaiah, P. Sathyanarayana, L. Guru Kumar," Image Texture Feature Extraction Using GLCM Approach", International Journal of Scientific and Research Publications, Volume 3, Issue 5, May 2013. 